\title{
Neurobehavioral Changes: An under looked aspect of Head Injury
}

\author{
Dr. Suman Rijal', Dr. Pankaj Raj Nepal ${ }^{2}$ \\ ${ }^{1}$ Department of Neurosurgery National Institute of Neurological and Allied Sciences Bansbari, Kathmandu, \\ Nepal \\ ${ }^{2}$ Department of Neurosurgery, B and C Medical College Teaching Hospital and Research Center, Birtamode, \\ Jhapa, Nepal.
}

\section{Correspondence:}

Dr. Suman Rijal, Associate Professor

Department of Neurosurgery, Upendra Devkota Memorial National Institute of Neurological and Allied Sciences, Bansbari, Kathmandu, Nepal.

Phone number: +977- 9841315945

Email: dr.rijalsuman@gmail.com

\begin{abstract}
Background: Different types of behavioral changes are seen in head injury patients, and these changes are directly or indirectly related to daily activities. Major alterations of personality after head injury are generally seen in the patients with severe head injury. However, disturbing post-concussional symptoms like headache, dizziness and memory problems generally persists for few months even in the less severely injured ones.

Materials and methods: Objective: To analyze the neurobehavioral changes in adult head injured patients. Study design: Prospective analytical study. Sampling technique: Non - probability consecutive sampling. Site of study: National Institute of Neurological and Allied Sciences, Bansbari, Nepal. Inclusion Criteria: All head injured patients above the age of 16 years. Exclusion Criteria: Extended Glasgow Outcome Scale of less than 3 at 6 months follow up. Data Collection and Analysis: All patients above the age of 16 years with head injury who got admitted were enrolled in the study. Parameters like age, gender, mode of injury, GCS at presentation were recorded. Extended Glasgow Outcome Scale along with Neurobehavioral rating was evaluated at 6 months. Then neurobehavioral rating scale was obtained by direct interview. Data analysis was done using SPSS v.20.

Results: Total number patients were 76 among which $71 \%$ were below 40 years of age and majorities $(87 \%)$ were males. Neurobehavioral categories like abnormal intentional behavior, lowered emotional state, heightened emotional state, arousal state and language had significant association with GCS at presentation and EGOS at 6 months. Similarly, age had significant association with language, where there was absent to mild language difficulty in patients below 40 years of age. Likewise, language difficulty, lower emotional state and abnormal intentional behavior were significantly associated with gender, as it was mild to severe in $30 \%$ of the female population who had sustained head injury.

Conclusion: Several neurobehavioral characters seem to be present in the various categories of the head injured patients in different ratios. Language problems seems to be less in the younger patients below the age of 40 years; although, few neurobehavioral parameters seems to affects the females more common compared to male counterparts. Also, family disruption and its extent of severity was significantly related to the severity of head injury
\end{abstract}

Key words: Extended Glasgow Outcome Scale, Head injury, Neurobehavioral Rating Scale, Traumatic brain injury.

$\mathrm{B}$

ehavioral disturbance is frequently manifested by patients during the subacute stage of recovery from severe closed head injury. ${ }^{1-7}$ Residual behavioral problems are particularly debilitating sequelae of severe closed head injury which contribute immensely to chronic disability, 8 impose a burden on the family ${ }^{9-11}$ and provide a challenge to rehabilitation. ${ }^{10}$ 
Although major alterations of personality after head trauma are generally confined to severe injury, ${ }^{2.9,12}$ post-concussional symptoms like headaches, dizziness, fatigability, difficulty in concentration and memory and associated emotional distress may frequently persist for at least 1 to 3 months following minor headinjury. ${ }^{13-}$ ${ }^{15}$ These symptoms, however minor, could nonetheless have long term psychosocial implications.

Administration of structured interviews and rating scales to relatives has elucidated the diversity of behavioral sequelae exhibited by head injured patients in various situations and the psychiatric repercussions imposed on family members., ${ }^{9,16}$ However, ratings by the clinician-investigator and self-reports by the patient can also be informative in assessing psychosocial outcome of closed head injury.

With the aim to analyze the neurobehavioral changes in adult head injured patients this study was performed.

\section{Methods and Materials:}

Analytical prospective study at National institute of Neurological and Allied Sciences Bansbari, Kathmandu, Nepal

Sampling technique: Consecutive non - probability sampling

Inclusion Criteria: All head injured patients above the age of 16 years. Exclusion Criteria: Extended Glasgow Outcome Scale of less than 3 at 6 months follow up.

Data Collection and Analysis: All patients who met the inclusion criteria were enrolled in the study. Their age, gender, mode of injury, GCS at presentation were collected. Extended Glasgow Outcome Scale and Neurobehavioral rating were evaluated at 6 months. Then neurobehavioral rating scale was obtained from all the remaining patients by direct interview using Levin's Neurobehavioral Rating (NBR) Scale - Revised. ${ }^{17}$ Data analysis was done using SPSS v.20. The frequencies were calculated for age, gender, mode of injury, severity of head injury, GOS-E and NBR changes.
Similarly, chi square test or fisher exact test where applicable were used to see the association of age, gender, severity of head injury, and GOS-E with the different NBR categories.

\section{Results:}

Total number of 76 patients were enrolled in the study with male $(66,87 \%)$ predominance compared to females $(10,13 \%)$. The mean age of the study population was 34.15 (SD 14.76). Majority of the injuries were due to road traffic accident (RTA) (46\%), followed by injury due to fall (28\%), physical assault $(20 \%)$ and other mode of injuries $(6 \%)$. In this study, $76 \%$ were mild head injury, followed by severe head injury $(15 \%)$ and moderated head injury $(9 \%)$

GOS-E of eight was seen in $43 \%$ followed by GOS-E of seven in $37 \%$. However, there were $20 \%$ of patients with GOS-E of $\leq 6$.

Abnormal overall NBR in terms of abnormal intentional behavior, lowered emotional state, heightened emotional state, arousal state, and language were $10(13.4 \%), 10(13.4 \%), 10(13.4 \%)$, $8(10.5 \%)$, and $11(14.5 \%)$ respectively (Table 1$)$.

Table 1: Overall Neurobehavioral changes

\begin{tabular}{|c|c|c|c|c|c|}
\hline $\begin{array}{c}\text { Neurobeha } \\
\text { vioral } \\
\text { Changes }\end{array}$ & Absent & Mild & Moderate & Severe & $\begin{array}{c}\text { Total } \\
\text { abnormal } \\
\text { NBR }\end{array}$ \\
\hline $\begin{array}{l}\text { Abnormal } \\
\text { intentional } \\
\text { behavior }\end{array}$ & $66(86.8 \%)$ & $7(9.2 \%)$ & $2(2.6 \%)$ & $1(1.3 \%)$ & $\begin{array}{c}10 \\
(13.4 \%)\end{array}$ \\
\hline $\begin{array}{l}\text { Lowered } \\
\text { emotional } \\
\text { state }\end{array}$ & $66(86.6 \%)$ & $9(11.8 \%)$ & - & $1(1.3 \%)$ & $10(13.4 \%)$ \\
\hline $\begin{array}{l}\text { Heightened } \\
\text { emotional } \\
\text { state }\end{array}$ & $66(86.6 \%)$ & $7(9.2 \%)$ & $2(2.6 \%)$ & $1(1.3 \%)$ & $10(13.4 \%)$ \\
\hline $\begin{array}{l}\text { Arousal } \\
\text { state }\end{array}$ & $68(89.5 \%)$ & $6(7.9 \%)$ & $2(2.6 \%)$ & - & $8(10.5 \%)$ \\
\hline Language & $65(85.5 \%)$ & $10(13.2 \%)$ & - & $1(1.3 \%)$ & $11(14.5 \%)$ \\
\hline
\end{tabular}

However, sub-categorical analysis of individual behavior showed $34 / 76$ had headache as a somatic egneuro, Volume 03, Issue 01, 2021 
concern, followed by 22/76 had depressive mood, $19 / 76$ had irritability, $18 / 76$ had agitation and so on (Table 2 and 3 )

Table 2: Overall frequency of different NBR category

\begin{tabular}{|c|c|c|c|c|c|}
\hline NBR individual & Absent & Mild & Moderate & Severe & $\begin{array}{l}\text { Total of } \\
\text { abnormal } \\
\text { NBR } \\
\text { Category }\end{array}$ \\
\hline \multicolumn{6}{|c|}{ Intentional Behavior } \\
\hline $\begin{array}{l}\text { Decreased } \\
\text { initiative or } \\
\text { motivation }\end{array}$ & 65 & 7 & 3 & 1 & 11 \\
\hline Blunted affect & 62 & 11 & 2 & 1 & 14 \\
\hline $\begin{array}{l}\text { Difficulty in } \\
\text { planning }\end{array}$ & 64 & 7 & 4 & 1 & 12 \\
\hline $\begin{array}{l}\text { Conceptual } \\
\text { disorganization }\end{array}$ & 66 & 5 & 4 & 1 & 10 \\
\hline $\begin{array}{l}\text { Self-appraisal } \\
\text { difficulties }\end{array}$ & 64 & 8 & 3 & 1 & 12 \\
\hline Disorientation & 63 & 9 & 3 & 1 & 13 \\
\hline $\begin{array}{l}\text { Difficulty in } \\
\text { mental flexibility }\end{array}$ & 66 & 8 & 1 & 1 & 10 \\
\hline $\begin{array}{l}\text { Memory } \\
\text { difficulties }\end{array}$ & 62 & 10 & 3 & 1 & 14 \\
\hline \multicolumn{6}{|c|}{ Emotional State (Lowered) } \\
\hline Depressive mood & 54 & 20 & 1 & 1 & 22 \\
\hline Anxiety & 60 & 15 & 0 & 1 & 16 \\
\hline $\begin{array}{l}\text { Emotional } \\
\text { withdrawal }\end{array}$ & 66 & 7 & 2 & 1 & 10 \\
\hline
\end{tabular}

Table 3: Overall frequency of different NBR category contd...

\begin{tabular}{cccccc}
\hline NBR individual & Absent & Mild & Moderate & Severe & $\begin{array}{c}\text { Total of } \\
\text { abnormal } \\
\end{array}$ \\
& & & & NBR \\
& & & & Category \\
& & & & \\
\hline
\end{tabular}

Survival Orientation Behavior/ Emotional state (heightened) :

\begin{tabular}{|c|c|c|c|c|c|}
\hline Irritability & 57 & 12 & 5 & 2 & 19 \\
\hline Hostility & 63 & 10 & 1 & 2 & 13 \\
\hline Disinhibition & 63 & 10 & 2 & 1 & 13 \\
\hline $\begin{array}{l}\text { Hyperactivity / } \\
\text { agitation }\end{array}$ & 58 & 10 & 7 & 1 & 18 \\
\hline $\begin{array}{l}\text { Unusual thought } \\
\text { content }\end{array}$ & 65 & 7 & 3 & 1 & 11 \\
\hline Excitement & 68 & 4 & 4 & 0 & 8 \\
\hline Suspiciousness & 74 & 0 & 1 & 1 & 2 \\
\hline Liability of mood & 62 & 10 & 3 & 1 & 14 \\
\hline \multicolumn{6}{|c|}{ Arousal State : } \\
\hline $\begin{array}{l}\text { Reduced } \\
\text { alertness }\end{array}$ & 66 & 9 & 1 & 0 & 10 \\
\hline $\begin{array}{l}\text { Mental } \\
\text { fatigability }\end{array}$ & 68 & 6 & 2 & 0 & 8 \\
\hline Attention & 66 & 9 & 1 & 0 & 10 \\
\hline Motor slowing & 65 & 8 & 2 & 1 & 11 \\
\hline \multicolumn{6}{|c|}{ Language : } \\
\hline $\begin{array}{l}\text { Difficulty in oral } \\
\text { expression }\end{array}$ & 62 & 11 & 2 & 1 & 14 \\
\hline $\begin{array}{l}\text { Difficulties in } \\
\text { oral } \\
\text { comprehension }\end{array}$ & 65 & 7 & 3 & 1 & 11 \\
\hline $\begin{array}{l}\text { Difficulty in } \\
\text { articulation }\end{array}$ & 66 & 8 & 2 & 0 & 10 \\
\hline
\end{tabular}

\begin{tabular}{lccccc}
\hline \multicolumn{5}{c}{ Not included in NRS -R item : } \\
\hline $\begin{array}{l}\text { Somatic concern } \\
\text { (headache) }\end{array}$ & 42 & 30 & 4 & 0 & 34 \\
Guilt & 70 & 6 & 0 & 0 & 6 \\
Hallucinations & 73 & 3 & 0 & 0 & 3 \\
\hline
\end{tabular}

While calculating the overall NBR abnormalities with the various head injuries grades, all of them 
were present in moderate and severe head injuries, which was 5(71.4\%) and $8(72.7 \%)$ respectively (Table 4).

Table 4: Overall NBR Abnormality in Various Head Injury Grades

\begin{tabular}{lcc}
\hline & $\begin{array}{c}\text { No. of } \\
\text { Patients }\end{array}$ & $\begin{array}{c}\text { Overall NBR } \\
\text { abnormality }\end{array}$ \\
\hline Mild Head Injury & 58 & $0(0 \%)$ \\
$\begin{array}{l}\text { Moderate Head } \\
\text { injury }\end{array}$ & 7 & $5(71.4 \%)$ \\
Severe Head injury & 11 & $8(72.7 \%)$ \\
Total & 76 & $13(17.1 \%)$ \\
\hline
\end{tabular}

However, sub-group analysis of each of the behavioral changes showed 24 out of 58 mild head injured patients had headache as a somatic concern followed by few other changes like depressive mood, anxiety and irritability.

Overall association of gender with NBR was not statistically significant; however, sub-group analysis showed females to be significantly more affected with Intentional behavior, Lowered emotional state, Language. Similarly males were more affected with heightened emotional behavioral changes significantly (Table 5).

Table 5: Sex Vs NBR in different category

\begin{tabular}{cccccc}
\hline \multicolumn{5}{c}{ Intentional behavior } \\
\hline Sex & Absent & Mild & Moderate & Severe & P Value \\
\hline Female & $7(70 \%)$ & $2(20 \%)$ & 0 & $1(10 \%)$ & $0.033^{*}$ \\
Male & 59 & 5 & 2 & 0 & \\
& $(89.39 \%)$ & $(7.58 \%)$ & $(3.03 \%)$ & & \\
\hline \multicolumn{7}{c}{ Lowered emotional state } \\
Female & $7(70 \%)$ & $2(20 \%)$ & 0 & $1(10 \%)$ & $0.022^{*}$ \\
Male & 59 & 7 & 0 & 0 & \\
& $(89.39 \%)$ & $(10.61 \%)$ & & &
\end{tabular}

Heightened emotional state

\begin{tabular}{|c|c|c|c|c|c|}
\hline Female & $9(90 \%)$ & 0 & 0 & $1(10 \%)$ & $0.046^{*}$ \\
\hline Male & $\begin{array}{c}57 \\
(86.36 \%)\end{array}$ & $\begin{array}{c}7 \\
(10.61 \%)\end{array}$ & $\begin{array}{c}2 \\
(3.03 \%)\end{array}$ & 0 & \\
\hline \multicolumn{6}{|c|}{ Arousal state } \\
\hline Female & $8(80 \%)$ & $1(10 \%)$ & $1(10 \%)$ & 0 & 0.279 \\
\hline Male & $\begin{array}{c}60 \\
(90.91 \%)\end{array}$ & $\begin{array}{c}5 \\
(7.58 \%)\end{array}$ & $\begin{array}{c}1 \\
(1.52 \%)\end{array}$ & 0 & \\
\hline \multicolumn{6}{|c|}{ Language } \\
\hline Female & $7(70 \%)$ & $2(20 \%)$ & 0 & $1(10 \%)$ & $0.026 *$ \\
\hline Male & $\begin{array}{c}58 \\
(87.88 \%)\end{array}$ & $\begin{array}{c}8 \\
(12.12 \%)\end{array}$ & 0 & 0 & \\
\hline
\end{tabular}

Overall association of age with NBR was not statistically significant; however, sub-group analysis showed age group of 60-69 year is more affected in language (Table 6 and 7).

Table 6: Age Vs NBR category

\begin{tabular}{|c|c|c|c|c|c|}
\hline \multicolumn{6}{|c|}{ Intentional behavior } \\
\hline Age & Absent & Mild & Moderate & Severe & P Value \\
\hline $10-19$ & $13(92.86 \%)$ & 0 & 0 & $\begin{array}{c}1 \\
(7.14 \%)\end{array}$ & 0.399 \\
\hline $20-29$ & $15(78.95 \%)$ & $4(21.05 \%)$ & 0 & 0 & \\
\hline 30-39 & $19(90.48 \%)$ & $1(4.76 \%)$ & $1(4.76 \%)$ & 0 & \\
\hline $40-49$ & $7(87.5 \%)$ & 0 & $1(12.55)$ & 0 & \\
\hline $50-59$ & $9(90 \%)$ & $1(10 \%)$ & 0 & 0 & \\
\hline $60-69$ & $3(75 \%)$ & $1(25 \%)$ & 0 & 0 & \\
\hline \multicolumn{6}{|c|}{ Lowered emotional state } \\
\hline $10-19$ & $12(85.71 \%)$ & $1(7.14 \%)$ & 0 & $\begin{array}{c}1 \\
(7.14 \%)\end{array}$ & 0.284 \\
\hline $20-29$ & $16(84.21 \%)$ & $3(15.79 \%)$ & 0 & 0 & \\
\hline 30-39 & $19(90.48 \%)$ & $2(9.52 \%)$ & 0 & 0 & \\
\hline $40-49$ & $7(87.5 \%)$ & $1(12.55)$ & 0 & 0 & \\
\hline
\end{tabular}

egneuro, Volume 03, Issue 01, 2021 


\begin{tabular}{ccccc}
$\mathbf{5 0 - 5 9}$ & $10(100 \%)$ & 0 & 0 & 0 \\
$60-69$ & $2(50 \%)$ & $2(50 \%)$ & 0 & 0 \\
\hline
\end{tabular}

Table 7: Age Vs NBR category contd...

\begin{tabular}{|c|c|c|c|c|c|}
\hline \multicolumn{6}{|c|}{ Heightened emotional state } \\
\hline $10-19$ & $12(85.71 \%)$ & $1(7.14 \%)$ & 0 & $\begin{array}{c}1 \\
(7.14 \%)\end{array}$ & 0.183 \\
\hline $20-29$ & $17(89.47 \%)$ & $\begin{array}{c}2 \\
(10.53 \%)\end{array}$ & 0 & 0 & \\
\hline 30-39 & $19(90.48 \%)$ & 0 & $\begin{array}{c}2 \\
(9.52 \%)\end{array}$ & 0 & \\
\hline $40-49$ & $7(87.5 \%)$ & $1(12.55)$ & 0 & 0 & \\
\hline 50-59 & $9(90 \%)$ & $1(10 \%)$ & 0 & 0 & \\
\hline $60-69$ & $2(50 \%)$ & $2(50 \%)$ & 0 & 0 & \\
\hline \multicolumn{6}{|c|}{ Arousal state } \\
\hline $10-19$ & $13(92.86 \%)$ & 0 & $\begin{array}{c}1 \\
(7.14 \%)\end{array}$ & 0 & 0.169 \\
\hline $20-29$ & $18(94.74 \%)$ & $1(5.26 \%)$ & 0 & 0 & \\
\hline 30-39 & $19(90.48 \%)$ & $1(4.76 \%)$ & $\begin{array}{c}1 \\
(4.76 \%)\end{array}$ & 0 & \\
\hline $40-49$ & $7(87.5 \%)$ & $1(12.55)$ & 0 & 0 & \\
\hline 50-59 & $9(90 \%)$ & $9(90 \%)$ & 0 & 0 & \\
\hline $60-69$ & $2(50 \%)$ & $2(50 \%)$ & 0 & 0 & \\
\hline \multicolumn{6}{|c|}{ Language } \\
\hline 10-19 & $13(92.86 \%)$ & $1(7.14 \%)$ & 0 & 0 & $0.022 *$ \\
\hline $20-29$ & $15(78.95 \%)$ & $\begin{array}{c}4 \\
(21.05 \%)\end{array}$ & 0 & 0 & \\
\hline 30-39 & $19(90.48 \%)$ & $2(9.52 \%)$ & 0 & 0 & \\
\hline
\end{tabular}

$\begin{array}{ccccc}\mathbf{4 0 - 4 9} & 7(87.5 \%) & 1(12.55) & 0 & 0 \\ \mathbf{5 0 - 5 9} & 9(90 \%) & 1(10 \%) & 0 & 0 \\ \mathbf{6 0 - 6 9} & 2(50 \%) & 1(25 \%) & 0 & 1(25 \%)\end{array}$

The association of admission GCS has got significant relation with the overall neurobehavioral changes (P Value $<0.001 *$ ). The more the grade of head injury the more is its association with the NBR changes (Table 8).

Table 8: Grade of head injury Vs NBR category

\begin{tabular}{|c|c|c|c|c|c|}
\hline \multicolumn{6}{|c|}{ Intentional behavior } \\
\hline GCS & Absent & Mild & Moderate & Severe & P Value \\
\hline \multicolumn{6}{|c|}{ Category } \\
\hline 13-15 & $58(100 \%)$ & 0 & 0 & 0 & $0.000^{*}$ \\
\hline 9-12 & $3(42.86 \%)$ & $2(28.57 \%)$ & $1(14.29 \%)$ & $1(14.29 \%)$ & \\
\hline 3-8 & $5(45.45 \%)$ & $5(45.45 \%)$ & $1(9.09 \%)$ & 0 & \\
\hline \multicolumn{6}{|c|}{ Lowered emotional state } \\
\hline 13-15 & $58(100 \%)$ & 0 & 0 & 0 & $0.000^{*}$ \\
\hline 9-12 & $4(57.14 \%)$ & $2(28.57 \%)$ & 0 & $1(14.29 \%)$ & \\
\hline 3-8 & $4(36.36 \%)$ & $7(63.64 \%)$ & 0 & 0 & \\
\hline \multicolumn{6}{|c|}{ Heightened emotional state } \\
\hline 13-15 & $58(100 \%)$ & 0 & 0 & 0 & $0.000^{*}$ \\
\hline $9-12$ & $2(28.57 \%)$ & $\begin{array}{c}4 \\
(57.14 \%)\end{array}$ & 0 & $1(14.29 \%)$ & \\
\hline 3-8 & $6(54.55 \%)$ & $3(27.27 \%)$ & $2(18.18 \%)$ & 0 & \\
\hline \multicolumn{6}{|c|}{ Arousal state } \\
\hline 13-15 & $58(100 \%)$ & 0 & 0 & 0 & $0.000^{*}$ \\
\hline $9-12$ & $3(42.86 \%)$ & $\begin{array}{c}3 \\
(42.86 \%)\end{array}$ & 0 & $1(14.29 \%)$ & \\
\hline 3-8 & $7(63.64 \%)$ & $\begin{array}{c}3 \\
(27.27 \%)\end{array}$ & 0 & $1(14.29 \%)$ & \\
\hline
\end{tabular}

Language 


\begin{tabular}{lccccc}
\hline 13-15 & $58(100 \%)$ & 0 & 0 & 0 & $0.000^{*}$ \\
9-12 & $2(28.57 \%)$ & 5 & 0 & 0 & \\
& & $(71.43 \%)$ & & & \\
3-8 & $5(45.45 \%)$ & $5(45.45 \%)$ & 0 & $1(9.09 \%)$ & \\
\hline
\end{tabular}

The association of GOS-E has got significant relation with the overall neurobehavioral changes (P Value $<0.001^{*}$ ). The low the GOS-E score, higher is the frequency of the abnormal NBR noted and was significant in all the sub-group categories (Table 9 and 10).

Table 9: Relation of GOS-E with NBR category at 6 months

\begin{tabular}{|c|c|c|c|c|c|}
\hline \multicolumn{6}{|c|}{ Intentional behavior } \\
\hline $\begin{array}{l}\text { GOS-E } \\
\text { at } \\
\text { 6month }\end{array}$ & Absent & Mild & Moderate & Severe & $\begin{array}{c}\mathbf{P} \\
\text { Valu } \\
\mathbf{e}\end{array}$ \\
\hline 4 & $1(33.33 \%)$ & $1(33.33 \%)$ & 0 & $1(33.33 \%)$ & $\begin{array}{c}0.00 \\
0^{*}\end{array}$ \\
\hline 5 & $3(27.27 \%)$ & $6(54.54 \%)$ & $2(18.18 \%)$ & 0 & \\
\hline 6 & $1(100 \%)$ & 0 & 0 & 0 & \\
\hline 7 & $28(100 \%)$ & 0 & 0 & 0 & \\
\hline 8 & $33(100 \%)$ & 0 & 0 & 0 & \\
\hline \multicolumn{6}{|c|}{ Lowered emotional state } \\
\hline \multicolumn{6}{|c|}{ GOS-E at 6month } \\
\hline 4 & 0 & $2(66.67 \%)$ & 0 & $1(33.33 \%)$ & $\begin{array}{c}0.00 \\
0^{*}\end{array}$ \\
\hline 5 & $4(36.36 \%)$ & $7(63.64 \%)$ & 0 & 0 & \\
\hline 6 & $1(100 \%)$ & 0 & 0 & 0 & \\
\hline 7 & $28(100 \%)$ & 0 & 0 & 0 & \\
\hline 8 & $33(100 \%)$ & 0 & 0 & 0 & \\
\hline
\end{tabular}

Heightened emotional state

GOS-E at 6month

\begin{tabular}{cccccc}
\hline $\mathbf{4}$ & $1(33.33 \%)$ & $1(33.33 \%)$ & 0 & $1(33.33 \%)$ & 0.00 \\
& & & & 0 \\
$\mathbf{5}$ & $4(36.36 \%)$ & $5(45.45 \%)$ & $2(18.18 \%)$ & 0 & \\
$\mathbf{6}$ & $1(100 \%)$ & 0 & 0 & 0 & \\
$\mathbf{7}$ & $28(100 \%)$ & 0 & 0 & 0 & \\
$\mathbf{8}$ & $32(96.96 \%)$ & $1(3.04 \%)$ & 0 & 0 & \\
\hline
\end{tabular}

Table 10: Relation of GOS-E with NBR category at 6 months contd...

\section{Arousal state}

\section{GOS-E at 6month}

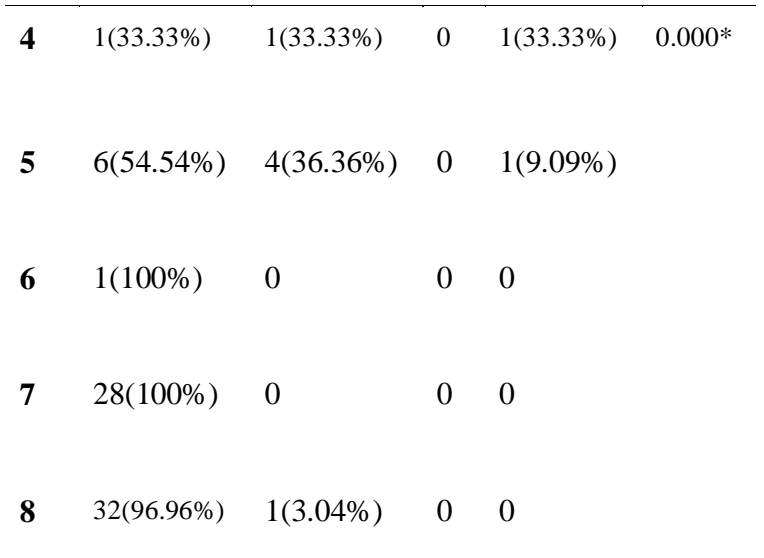

\section{Language}

GOS-E at 6month

\begin{tabular}{llllll}
\hline 4 & $1(33.33 \%)$ & $1(33.33 \%)$ & 0 & $1(33.33 \%)$ & $0.000^{*}$ \\
$\mathbf{5}$ & $3(27.27 \%)$ & $8(72.72 \%)$ & 0 & 0 & \\
$\mathbf{6}$ & $1(100 \%)$ & 0 & 0 & 0 \\
7 & $28(100 \%)$ & 0 & 0 & 0
\end{tabular}




\section{Discussion:}

In this study, abnormal overall NBR in terms of abnormal intentional behavior, lowered emotional state, heightened emotional state, arousal state, and language were 10 (13.4\%), 10(13.4\%), 10(13.4\%), $8(10.5 \%)$, and $11(14.5 \%)$ respectively. However, sub-categorical analysis of individual behavior showed 34/76 had headache as a somatic concern, followed by $22 / 76$ depressive mood, 19/76 irritability, 18/76 agitation and so on. Similarly, in the study of Mazaux JM et al18 where they noted depressive mood being the most common $(52 \%)$ findings in the head injured patients, followed by mental fatigability (47\%), memory difficulty $(46 \%)$, anxiety (28\%), lack of initiative and motivation $(28 \%)$ and so on. These findings are somewhat similar to this present study.

In the literatures, neurobehavioral abnormalities were more severe in patients with moderate and severe head injuries compared to the mild head injury. ${ }^{17}$ In our study, $71.4 \%$ of the moderate and $72.7 \%$ of the severe head injured patients had such abnormality. The somatic concern like headache were present in 24 out of 58 mild head injured patients followed by few other changes like depressive mood, anxiety and irritability in the same head injury category. The grade of head injuries were also highly significant in all the categories of NBR, where poor the initial GCS had the worst neurobehavioral abnormalities.

Also as expected, better the outcome of the patients at six months, there was less severe the NBR and these values were also highly significant in all the subgroup analysis of the NBR.

Gender is not often tested against the occurrence of neurobehavioral changes. However, in our study, sub-group analysis showed females to be significantly more affected with Intentional behavior, Lowered emotional state and Language. Males were however; more affected with Heightened emotional behavioral changes significantly.
Age could be another confounding variable in the neurobehavioral changes, which in our study showed occurrence of language problem in the elderly population ( $>60$ years).

\section{Conclusion:}

On the basis of this modest study if we may, we like to suggest that neurobehavioral changes seems to be found in all grades of severity with the severe ones having the highest. Females seem to be more affected with abnormality in intentional behavior, lowered emotional state and language whereas males are more affected with heightened emotional state. Also, these neurobehavioral changes seem to be strongly associated with admission GCS and GOS-E.

\section{References:}

1. Levin HS, Grossman RG. Behavioral sequelae of closed head injury. A quantitative study. Arch Neurol 1978; 35:720-7.

2. Lishman WA. The psychiatric sequelae of head injury: a review. Psychol Med 1973;3: 304-18.

3. Ruesch J. Intellectual impairment in head injuries. Am J Psychiatry 1944; 100:480-96.

4. Ruesch J, Bowman KM. Prolonged post-traumatic syndromes following head injury. Am J Psychiatry1945; 102:145-63.

5. Schilder P. Psychic disturbances after head injuries. Am J Psychiatry 1934; 91:155-88.

6. Schnaper N. The psychological implications of severe trauma: emotional sequelae to unconsciousness. Apreliminary study. J Trauma 1975; 15:94-8.

7. Stem JM. Cranio-cerebral injured patients. A psychiatric clinical description. Scand J Rehab Med 1978; 10:7-10.

8. Jennett $B$ et al. Disability after severe head injury: observations on the use of the Glasgow Outcome Scale. J Neurol Neurosurg Psychiatry 1981; 44:285-93.

9. Brooks DN, McKinlay W. Personality and behavioural change after severe blunt head injury-a relative's view. J Neurol Neurosurg Psychiatry 1983; 46:336-44.

10. Fordyce DJ, Roueche JR, Prigatano GP. Enhanced emotional reactions in chronic head trauma patients. $\mathrm{J}$ Neurol Neurosurg Psychiatry 1983; 46:620-4.

11. Livingston MG, Brooks DN, Bond MR. Three months after severe head injury: psychiatric and social impact on relatives. J Neurol Neurosurg Psychiatry 1985; 48:870-5.

12. McKinlay WW et al. The short-term outcome of severe blunt head injury as reported by relatives of the injured persons. J Neurol Neurosurg Psychiatry 1981; 44:727-33.

13. Gronwall D, Wrightson P. Delayed recovery of intellectual function after minor head injury. Lancet 1974; ii: 605-9. 
14. Levin HS, Mattis S, Ruff RM. Neurobehavioraloutcome of minor head injury: a three center study. J Neurology.

15. Rutherford WH, Merrett JD, McDonald JR. Sequelaeof concussion caused by minor head injuries. Lancet 1977; i: $1-4$.

16. Weddell R, Oddy M, Jenkins D. Social adjustment after rehabilitation: a two year follow-up of patients with severe head injury. Psychol Med 1980; 10:257-63.

17. 5. Vanier M, Mazaux J-M, Lambert J, et al. Assessment of neuropsychologic impairments after head injury: interrater reliability and factorial and criterion validity of the neurobehavioral rating scale, revised. Arch Phys Med Rehabil 2000; 81:796-806.

18. 4. Mazaux JM, Masson F, Levin HS, Alaoui P, Maurette P, Barat M. Long-term nemopsychological outcome and loss of social autonomy after traumatic brain injury. Arch Phys Med Rehabil 1997; 78:1316-20. 